\title{
VARIOUS MATHEMATICAL APPROACHES TO EXTRACT INFORMATION FROM TEXTURES OF INCREASING COMPLEXITIES
}

\author{
Fahima Nekka ${ }^{1}$ and $\mathrm{Jun} \mathrm{Li}^{2}$ \\ 1 Faculté de Pharmacie and Centre de Recherches Mathématiques, Université de \\ Montréal C.P. 6128, Succ. Centre-ville, Montréal (Québec), Canada H3C 3J7 \\ fahima.nekka@umontreal.ca \\ 2 Faculté de Pharmacie and Centre de Recherches Mathématiques, Université de \\ Montréal C.P. 6128, Succ. Centre-ville, Montréal (Québec), Canada H3C 3J7 \\ li@crm. umontreal.ca
}

Summary. Dealing with several structures of different complexities, we adopt various strategies to extract information. The general idea is to find appropriate tools to analyze the variation of the corresponding autocorrelation functions. First, for homogeneous media under different conditions, we recover, in a statistical way, a relationship between porosity and the autocorrelation function. Then, for low-complexity textures, we exploit this relationship to extract complementary parameters from the autocorrelation function beyond porosity using spectral analysis. For fractal-like structures, we process them according to their porosity. For fat fractals, usually used as synthetic models of porous media, we combine the regularization dimension, a method proposed to estimate the curve variation, with the autocorrelation function. This leads to a more robust classification. For fractals of negligible porosity, such as fractals of non-integer dimension, we discuss how the method HMSF we developed serves as an original means to estimate the Hausdorff dimension and how it can be exploited to give complementary characteristic parameters.

\section{Introduction}

New information and measurement technologies give access to signals of exceedingly complex nature, making it demanding for more sophisticated data analysis to efficiently extract information beyond the scope of the traditional methods. For example, the design of synthetic polymers has been revolutionized by the new achievements in high-resolution, broad-mass-range spectrometry [1]. Wave propagation and scattering through porous media and highly ramified materials lead to (spatial) signals which can be considered as defined on fractal systems $[2,3]$. As a matter of fact, the obtained mass spectra, which 
carry out microstructure information of great impact on the macroscopic physical properties, can be very complex. A central concern when processing such complex data is to use tools that extract the maximum information with the least degeneracy. The autocorrelating process, expressed through the autocorrelation function is a classical mathematical method widely used in engineering and applied sciences [4]. It is a powerful process that accumulates and reorganizes intrinsic similarities hidden in a structure, allowing thus for a (pre)processing of the signal as well as its analysis. Fractal methods already proved to be efficient to quantify complex information based on existing similarities. The major use of fractal analysis is to measure this complexity through fractal dimensions and other related indexes. However, inadequacy of traditional methods when processing complex systems and the known limitations of popular fractal methods led us to investigate, in parallel, these two different ways of information processing, and to combine them in order to create more powerful and less degenerate methods $[6,7,8,9]$. This resulted in an extension of classical methods, making them applicable within a nowadays context knowledge and in a benefit of fractal analysis from these classical and very popular methods [7].

This paper is organized as follows. In Section 2, we propose several statistical models of homogeneous media from which we recover the relationship between porosity and the autocorrelation function. In Section 3, we propose complementary parameters to porosity by exploiting various complexities of the autocorrelation functions of the signals. For low-complexity textures, we propose a measure of departure from homogeneity. For fractal-like textures exhibiting power law properties, usually used as synthetic porous media, we show how it is possible to classify them by combining the autocorrelation function with the regularization dimension [11]. We also consider the case of non-trivial structures having zero porosity (thin fractals). For these structures, we use what we previously called Hausdorff measure spectrum function (HMSF), which generalizes the classical autocorrelation function and proves to be more sensitive and suitable to describe this kind of scattered objects $[6,7,8]$. In section 4 , we comment our proposed strategies and suggest some possibilities to pursue additional work in this direction.

\section{A Statistical Relationship Between Porosity and the Autocorrelation Function}

In signal processing, the autocorrelation function is often regarded as another aspect of a signal which facilitates its analysis. To characterize a porous structure, porosity is considered as one principal index reflecting its spatial occupancy. In this section, based on several statistical models of porous images, we discuss the relationship between their porosity and their corresponding autocorrelation function. 
Assume that a signal $S(x)$ is of finite energy, we have the simplest form of the autocorrelation function:

$$
r_{S}(t)=\int_{-\infty}^{\infty} S(x) S(x+t) d x
$$

This self crossing process sums all point-point similarities (by product) of the signal at distances $t$. Given the signal of an image set, the autocorrelation function is dedicated to measure the similarity in the set's geometric structure [4]. Though various forms of autocorrelation functions are widely used, this basic idea is always the same.

Study of porous media mainly involves porosity, which refers to the occupancy of the set, measured on samples having regular geometrical shapes. To facilitate porosity estimation, we generally select for the sampling, the familiar shapes such as cubes, cylinders, spheres, etc.

For a porous media of a large size, the concept of deterministic porosity is questionable. We can invoke two possible reasons: the boundary as well as the volume of the compartment where lives the porous media, generally having a complex geometrical form, are difficult to be determined or the measurement cannot be taken on the whole porous media. Thus, we have to consider the porosity in a statistical way. This can be done by a sampling process. The sampling is carried out in an arbitrary way and different values of porosity are obtained when different sampling sizes and positions are considered. Since each chosen sample is within a compartment of regular shape, it has a deterministic porosity value. Average of these values will tend to the true porosity of the whole porous media, guaranteed by the so-called large number theorem. In fact, we have two choices: to approximate the porosity of the whole porous structure by the porosity of the samples chosen large enough (which is generally difficult as explained above) or, alternatively, to take a large number of samples.

For a porous structure represented by a spatial signal, it turns out that porosity have a very close relationship to the autocorrelation function. This connection is not perceptible in a deterministic case (one sample), but will be clear when explained in the following statistical synthetic models.

\subsection{A Homogeneous Model of a Single Component Porous Media with Point-to-point Independence}

For the sake of simplicity, we consider the one-dimensional case. We also assume the homogeneous porous set $F$ to be hosted in the unit interval and defined as follows:

At the finest resolution, the proposed porous media $F$ can be considered to be composed of $n$ equal parts $[(i-1) / n, i / n], i=1, \cdots, n$ of the unit interval, where each part is associated with a binary random variable $X_{i}$ indicating the membership of the subinterval $[(i-1) / n, i / n]$. We can write 
$F=\bigcup_{i: X_{i}=1}[(i-1) / n, i / n]$ and $X_{i}$ is defined as:

$$
\mathbb{P}\left(X_{i}=1\right)=p, \quad \mathbb{P}\left(X_{i}=0\right)=1-p
$$

where $\mathbb{P}$ denotes the probability.

Suppose that any part $[(i-1) / n, i / n]$ is chosen in a random way, we further assume that the variables $X_{i}$ are i.i.d. for all $1 \leq i \leq n$.

Consider the normalized version $I(t)$ of the autocorrelation function of $F$ :

$$
I(t)=\frac{\int \mathcal{X}_{F}(x) \mathcal{X}_{F}(x+t) d x}{\int \mathcal{X}_{F}(x)^{2} d x}
$$

where $\mathcal{X}_{F}$ is the indicator function of $F: \mathcal{X}_{F}(x)=\sum_{i=1}^{n} X_{i} \mathcal{X}_{[(i-1) / n, i / n]}(x)$ (the value at each $i / n$ is treated in a natural way, i.e. it can only be 0 or 1$)$. The normalization is applied to assure that $I(0)=1$, which will facilitate our comparisons below.

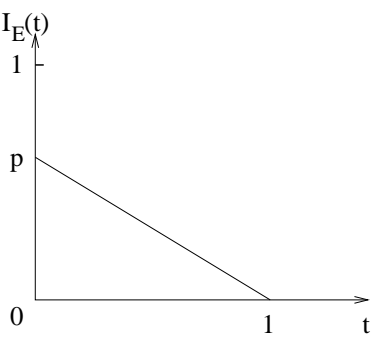

(a)

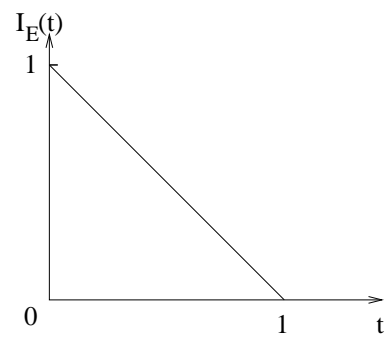

(b)

Fig. 1. The autocorrelation of: (a) the homogeneous model of single component porous media with point-to-point independence; (b) the unit segment.

In this example, we have

$$
\int \mathcal{X}_{F}(x)^{2} d x=\frac{\sum_{k=1}^{n} X_{k}^{2}}{n}
$$

and

$$
\int \mathcal{X}_{F}(x) \mathcal{X}_{F}(x+t) d x=\frac{\sum_{k=i}^{n} X_{k} X_{k-i+1}}{n}
$$

for $t=(i-1) / n, i=1, \cdots, n$.

Since we have assumed a statistical definition of $F$, it is suitable to redefine the autocorrelation function of $F$ in the following way:

$$
I_{\mathbb{E}}(t)=\frac{\mathbb{E} \int \mathcal{X}_{F}(x) \mathcal{X}_{F}(x+t) d x}{\mathbb{E} \int \mathcal{X}_{F}(x)^{2} d x}
$$


where $\mathbb{E}$ denotes the expectation.

Then, we have $\mathbb{E} \int \mathcal{X}_{F}(x) \mathcal{X}_{F}(x+t) d x=\left(1-\frac{i-1}{n}\right) p^{2}$ and $\mathbb{E} \int \mathcal{X}_{F}(x)^{2} d x=$

$p$. On the other hand, variances of these two expressions involved in $I_{\mathbb{E}}(t)$, are of order $1 / n$, i.e. $O(1 / n)$, which guarantees their convergence when $n$ is large enough.

It is easy to see that:

$$
I_{\mathbb{E}}(t)=\left\{\begin{aligned}
1, & t=0 ; \\
(1-(i-1) / n) p, t & =(i-1) / n, \quad i=2, \cdots, n .
\end{aligned}\right.
$$

Let $n$ go to infinity, we obtain:

$$
I_{\mathbb{E}}(t)= \begin{cases}1, & t=0 \\ (1-t) p, & 0<t<1 .\end{cases}
$$

Fig.1(a) illustrates $I_{\mathbb{E}}(t)$.

The porosity of $F$ is $\frac{1}{n} \sum_{i=1}^{n} X_{i}$; statistically, it is: $\frac{1}{n} \mathbb{E} \sum_{i=1}^{n} X_{i}=p$.

Then $p$ is obtained as the right limit of $I_{\mathbb{E}}(t)$ at $t=0$ or the slope of the $I_{\mathbb{E}}(t)$. In this way, the porosity of a set $F$ can be related to its autocorrelation function $I_{\mathbb{E}}(t)$ by considering an immediate neighborhood of $t=0$ or root mean square slope of the autocorrelation function.

The simple case of the autocorrelation function of the segment $[0,1]$, a limit case of Eq.(2.6), is illustrated in Fig.1(b). In this case, there's no jump from $t=0$ to $t=0+$, meaning that the similarity is carried out by all the points of $[0,1]$ and that $I_{\mathbb{E}}(t)$ smoothly decreases as $t$ increases. However, for $p<1$, from $t=0$ to $t=0+$, the similarity is carried out by only $p$ percent of points of $[0,1]$, which equals to the porosity of the set, Fig.1(a).

\subsection{A Homogeneous Model of Double Components Porous Media with Point-to-point Independence}

To verify the relationship between porosity and the autocorrelation function for a more complex structure, we study another model of porous media composed of two components.

Consider $F$ as composed from $2 n$ equal parts $\left[\frac{i-1}{2 n}, \frac{i}{2 n}\right], i=1, \cdots, 2 n$ of the unit interval, where each part is associated with a binary random variable $X_{i}$ indicating the membership of the subinterval $\left[\frac{i-1}{2 n}, \frac{i}{2 n}\right]$. Clearly, we can write $F=\bigcup_{i: X_{i}=1}\left[\frac{i-1}{2 n}, \frac{i}{2 n}\right]$ and $X_{i}$ is defined as:

$$
\mathbb{P}\left(X_{i}=1\right)=p_{j}, \quad \mathbb{P}\left(X_{i}=0\right)=1-p_{j},
$$

where $j=1$ for $i$ even, $j=2$ for $i$ odd, and $\mathbb{P}$ denotes the probability. 

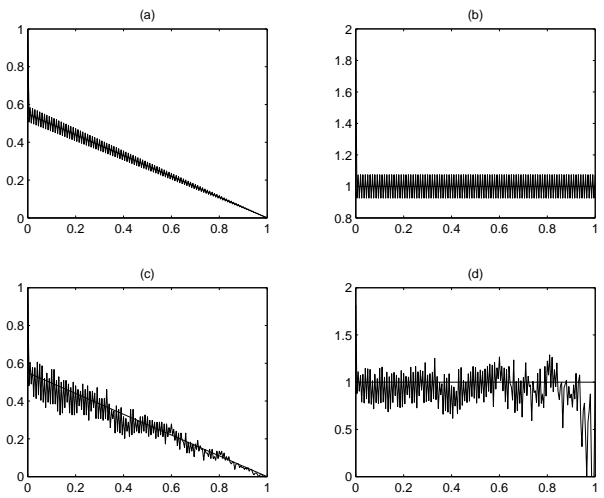

Fig. 2. The autocorrelation of porous media where $p_{1}=0.7, p_{2}=0.4$. Theoretical expressions: (a) $I_{\mathbb{E}}(t)$, (b) $I_{\mathbb{E}}(t) / I_{\mathbb{E}, p}(t)$; simulation results $(10,000$ samples $)$ : (c) $I_{\mathbb{E}}(t)$, $(\mathrm{d}) I_{\mathbb{E}}(t) / I_{\mathbb{E}, p}(t)$.

We further assume that the variables $X_{i}$ are independent between them, for all $1 \leq i \leq 2 n$. Then we have $\mathbb{E} \int \mathcal{X}_{F}(x)^{2} d x=\left(p_{1}+p_{2}\right) / 2$, which is in fact the statistical porosity of $F$, and we note $p=\left(p_{1}+p_{2}\right) / 2$. Moreover, we have:

$$
\mathbb{E} \int \mathcal{X}_{F}(x) \mathcal{X}_{F}(x+t) d x=\left(1-\frac{k}{n}\right)\left(p_{1}^{2}+p_{2}^{2}\right)
$$

for $t=\frac{2 k}{2 n}$, and

$$
\mathbb{E} \int \mathcal{X}_{F}(x) \mathcal{X}_{F}(x+t) d x=\left(1-\frac{2 k-1}{2 n}\right) p_{1} p_{2}
$$

for $t=\frac{2 k-1}{2 n}$. On the other hand, variances of these two expressions, involved in $I_{\mathbb{E}}(t)$, are $O(1 / n)$, which guarantees their convergence when $n$ is large enough. Then:

$$
I_{\mathbb{E}}(t)= \begin{cases}1, & t=0 ; \\ \left(1-\frac{2 k}{2 n}\right) \frac{p_{1}^{2}+p_{2}^{2}}{p_{1}+p_{2}}, \quad t=\frac{2 k}{2 n}, \quad k=1, \cdots, n ; & \frac{2 k+1}{2 n}, \quad k=1, \cdots, n .\end{cases}
$$

Consider the line of porosity defined by:

$$
I_{\mathbb{E}, p}(t)=(1-t) p, \quad 0<t<1 .
$$

Then, for $t=\frac{2 k}{2 n}, \quad k=1, \cdots, n$, we have: $I_{\mathbb{E}}(t)=(1-t) p \cdot \frac{4 p_{1} p_{2}}{\left(p_{1}+p_{2}\right)^{2}}<$ $I_{\mathbb{E}, p}(t)$, and for $t=\frac{2 k+1}{2 n}, \quad k=1, \cdots, n$, we have: $I_{\mathbb{E}}(t)=(1-t) p$. $\frac{2\left(p_{1}^{2}+p_{2}^{2}\right)}{\left(p_{1}+p_{2}\right)^{2}}>I_{\mathbb{E}, p}(t)$. 
Clearly, $I_{\mathbb{E}}(t)$ oscillates around $I_{\mathbb{E}, p}(t)$, making the latter as its line of average. In Fig. 2, we show the theoretical and simulation results for $I_{\mathbb{E}}(t)$ and $I_{\mathbb{E}}(t) / I_{\mathbb{E}, p}(t)$. We observe that the oscillations of the autocorrelation curve around its line of porosity will not vanish, reflecting the dependency between points. Also, the simulations indicate that the part of $I_{\mathbb{E}}(t) / I_{\mathbb{E}, p}(t)$ that can be used should not be close to $t=1$ since the divider will be too small.

\subsection{A Homogeneous Model of Porous Media with Point-to-point Correlation in Terms of Distance}

A more realistic model is to suppose some relationships between the points, i.e. a certain dependency between components. This dependency can be a function of the distance between points, and logically, it should decrease as the distance increases.

In this model, we consider the porous media as composed of one component, and we will keep all notations used in Section 2.1. We also have:

$$
\mathbb{E}\left(X_{i} X_{j}\right)= \begin{cases}p, & i=j \\ R(i, j) p, & i \neq j\end{cases}
$$

where $R(i, j)$ is a function of $|i-j|$ that can be redefined as $R(r), r=$ $|i-j| / n$.

$R(r)$ is positive and its largest value is $R(0)=1$. Moreover, $R(r)$ will oscillate around $p$ and converges to $p$ when $r$ increases and $R(+\infty)=p$.

We have

$$
\mathbb{E} \int \mathcal{X}_{F}(x)^{2} d x=\frac{1}{n} \sum_{k=1}^{n} \mathbb{E} X_{i}^{2}=p .
$$

and for $t=(i-1) / n$,

$$
\begin{aligned}
\mathbb{E} \int \mathcal{X}_{F}(x) \mathcal{X}_{F}(x+t) d x & =\frac{1}{n} \sum_{k=i}^{n} \mathbb{E}\left(X_{k} X_{k-i+1}\right) \\
& =(1-t) R(t) p
\end{aligned}
$$

Then

$$
I_{\mathbb{E}}(t)=(1-t) R(t) .
$$

Rewrite $I_{\mathbb{E}}(t)=R_{p}(t) R_{o}(t)$, where $R_{p}(t)=p(1-t)$ and $R_{o}(t)=R(t) / p$. This decomposition describes the two components that are present in the autocorrelation function. Indeed, $R_{p}$ is a straight line with slope $-p$ and cutting point $p$ on the $y$-axis. This line, that we called the line of porosity in Section 2.2, corresponds to the autocorrelation function of the point-to-point independent homogeneous porous media. The curve $R_{o}$ is the pure correlation component which contains the correlation information between points. In other terms, $R_{o}$ gives the information about the average relationship between 
any two points of the set in terms of their distance. Thus, we can redefine $R_{o}$ as the pure correlation function of the set:

$$
R_{o}(t)=\frac{\mathbb{E} \int \mathcal{X}_{F}(x) \mathcal{X}_{F}(x+t) d x}{(1-t) \mathbb{E} \int \mathcal{X}_{F}(x)^{2} d x} .
$$

Remark: The following autocorrelation function is commonly used for stationary signals $S$ :

$$
C(t)=\frac{E[(S(x)-m)(S(x+t)-m)]}{E(S(x)-m)^{2}}
$$

where $m=E(S(x))$ is independent of $x$.

However, for a spatial signal defined on a complex support, it is difficult or even impossible to define its mean value $m$, when taken in the usual meaning [5]. In order to use the last mentioned definition of the autocorrelation function, some ergodic condition on average has to be verified. To avoid this average problem, we chose to use the general form [4]. Moreover, as shown above, the general form can be decomposed, in a clear way, into two components that we call here the line of porosity and the pure correlation curve.

\section{Complementary Parameters to Porosity}

Obviously, porosity alone is far from enough to reflect the irregular morphology of micro-porous structures. Properties of porous media are highly dependent on the morphology of the pore space as well as those of its complementary part. To go one step further in the characterization process and extract complementary parameters, various strategies have to be developed for different degrees of complexity.

\subsection{Low-complexity Texture: A Measure of the Departure from Homogeneity}

Simply organized structures are generally composed of sub-parts having a narrow range of size distribution. Thread-like textures are a particular case (Fig. 3 , left and middle parts). In this part, we use typical thread-like structures to show how, our newly developed analysis approach, based on the autocorrelation function, allows to extract pore frequency and extent, which are complementary parameters to porosity. From the previous result, we know that the LMS of the autocorrelation function of a perfectly homogeneous structure coincides with its porosity line. This ideal homogeneous structure will serve to measure the departure of other structures from homogeneity. Comparing images based on this departure from homogeneity, based on the autocorrelation function, is more feasible than a direct pixel-by-pixel comparison. To do so, we suggest to subtract, form the autocorrelation function, its porosity line, which is, as stated above, its LMS slope. This allows to keep only the 


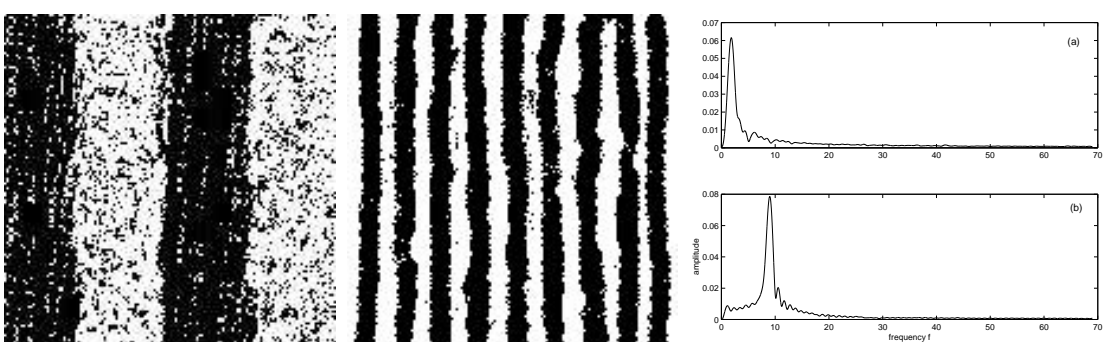

Fig. 3. Left and middle: two synthetic thread-like porous structures of same porosity; Right: once porosity line cleared, the Fourier transform of the autocorrelation functions, (a) for the left image, (b) for the middle one.

information hidden in the remaining part, $I_{\mathbb{E}}(t)-R_{p}$. In fact, this departure from homogeneity can be related to the concept of lacunarity [12]. For this remaining curve, two different approaches can be adapted. The first one is through the variance of $I_{\mathbb{E}}(t)-R_{p}$, which can be defined as a new formula for lacunarity as a departure from homogeneity, in terms of the autocorrelation function. This approach is not developed in this paper. Since we are concerned, in this section, by low-complexity images, it is possible to apply the Fourier transform to analyze the frequency components of $I_{\mathbb{E}}(t)-R_{p}$.

For example, for the two periodic patterns of the same porosity value: 0.4633, shown by two images on the left and middle places in Fig.3, the Fourier transform of their corresponding $I_{\mathbb{E}}(t)-R_{p}$ is given on the right of Fig.3. The results on frequency $f$ as well as on $1 / f$ give two parameters, independent from porosity, representing the number of threads as well as their sizes, respectively. In fact, the Fourier transform of $I_{\mathbb{E}}(t)-R_{p}$ gives a frequency expression of the departure of the signal from homogeneity (Wiener-Khinchin theorem). The main frequency component corresponds to periodic pattern once the porosity information is dropped.

We have also processed images where two frequencies have been superposed. To extract the average frequency $f_{m}$ and the corresponding average length $1 / \lambda_{m}$, we experimentally found that the following formula gives the nearest values for $f_{m}$ compared to $1 / \lambda_{m}$. If we note by $\mathcal{D}(f)$ the absolute value of Fourier transform of $I_{\mathbb{E}}(t)-R_{p}$, then, we have:

$$
\begin{aligned}
f_{m} & =\left(\frac{\sum \mathcal{D}\left(f_{i}\right) f_{i}^{1 / 2}}{\sum \mathcal{D}\left(f_{i}\right)}\right)^{2} \\
\lambda_{m} & =\left(\frac{\sum \mathcal{D}\left(f_{i}\right) \lambda_{i}^{1 / 2}}{\sum \mathcal{D}\left(f_{i}\right)}\right)^{2}
\end{aligned}
$$

where $\lambda_{i}=1 / f_{i}$.

We have to stress that the above Fourier analysis method works well for non-trivial cases, i.e. porosity is not too small or not near 1 (its complementary 

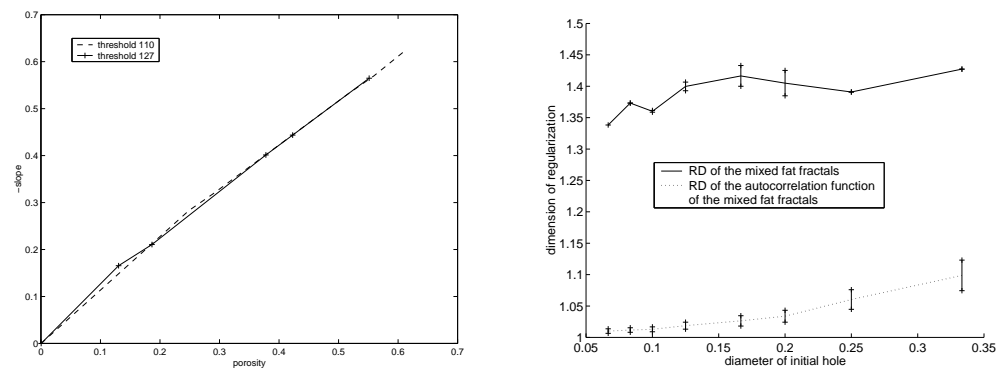

Fig. 4. Left: The matching between estimated porosity using the porosity formula and the slope of the linear part of the autocorrelation function of images in [13], page 118; Right: RD directly applied on the mixed fat fractals (top) and on their autocorrelation function (bottom).

is small). For complex structures of porosity near zero, we have to use the approaches we present below.

Remark: Relying on the theoretical proof given in Section 2, which gives a statistical meaning to porosity through autocorrelation function, we suggest to extract images porosity using the least mean square (LMS) slope of the autocorrelation function. This porosity line gives a statistical information on a given (deterministic) image, which, in fact, has been generated through a random way. This statistical meaning of porosity is more appropriate than using the porosity computed from a single image, the latter introducing a variation in the porosity of the whole images collection. As a practical example, we use a set of porous images of decreasing porosities to illustrate this correspondence of porosity to the LMS slopes of the autocorrelation function. The images are taken from K. Zhao et al. [13], Fig. 2 on page 118, which represent blending films of porous scaffold and show decreasing porosities in terms of the concentration of the two used polymers, PHB and PHBHHx. The left plot in Fig. 4 reports the equivalence (matching) between the LMS slopes of the autocorrelation of figures (a-f)in Fig. 2 in K. Zhao et al. [13] and the porosity values calculated by the porosity formula.

\subsection{Fractal-like Texture}

\section{Fat Fractals: Synthetic Porous Media}

When complexity of a structure increases further while it has a positive (nonzero) porosity, we propose a different approach to the autocorrelation function, combining fractal tools. A typical case for those more complex structures of positive porosity are known as fat fractals. Their main peculiarity, compared to thin fractals, consists in a finite and non-zero Lebesgue measure of their support. The empty holes of fat fractals have size-dependant power distribution similar to porous materials $[3,14]$ and they have been proposed as realistic models of micro-porous media [3], Let us recall how a 1-D regular 
fat fractal is generated. For sake of simplicity, we usually use the unit interval as initiator. A regular fat fractal can be obtained through the following simple iterative rule: from one step to the next, we just drop the open middle intervals of length $l_{n}$ from each of the remaining intervals. If the length of the interval at step $n-1$ is $L_{n-1}$, then this removed length is $L_{n-1}$ divided by $a^{n}$. After $n$ iterations, the obtained set is composed of $2^{n}$ intervals of lengths $L_{n}$ and $n$ subsets which are composed of $N_{n}(k)=2^{k-1}$ empty holes of lengths $l_{k},(k=1, \cdots, n)$, respectively. For a 1-D regular fat fractal, $a$ is the only parameter involved in its definition. It is clear that the empty holes are symmetrically distributed in a 1-D regular fat fractal. To describe a porous media having asymmetric features, we have to use the mixed fat fractal which, intuitively, is a redistribution of the parts of the regular one, obtained by rearranging alternatively its voids and occupied intervals. We have considered eight fat fractals having the following values for the parameter $a: 3,4,5,6$, $8,10,12$ and 15. The autocorrelation function of these fat fractals is a more irregular curve, preventing thus a valuable application of the Fourier transform as was the case above [15, 9]. The irregularity of those curves suggests the utility of the fractal dimension usually used to quantify complexity. Thus, we use the regularization dimension (RD), introduced by J. Levy-vehel and F. Roueff [11], since it proved to be more sensitive to variations. However, one can also raise the question of applying the $\mathrm{RD}$ directly to the fat fractal instead of applying it to the autocorrelation function of the set. Comparison of the obtained results shows that, when RD is directly applied to the sets, a differentiation based on this dimension is less convincing compared to when it is applied to their autocorrelation function, the right plot of Fig. 4. In fact, $\mathrm{RD}$ of the autocorrelation function is a strictly increasing function of the size of the initial hole. This difference can be explained by the fact that autocorrelation function has a smoothing "action" since it attenuates the irregularities of a signal (spatial signal representing the set in this case) and produces more uniform ones: sparse parts intersecting with themselves will still be sparse and when intersecting with denser parts, will again produce sparse parts. Also, the autocorrelation accumulates similarities that are dispersed all along the set. Once the autocorrelation function is applied, The similarity amount is globally decreasing as the translation value $t$ increases.

\section{Thin Fractals: Structures of Porosity Zero}

As already mentioned in Section 3.1, images of neglected porosity cannot be processed by the Fourier analysis approach that we suggested above. We hereafter propose an alternative method based on the Hausdorff measure of these sets. In fact, typical non-trivial examples are sets having zero-Lebesgue measure, as it is the case for Cantor sets or, more generally thin fractals. For these sets, we have to use what we previously named the Hausdorff Measure Spectrum Function (HMSF)[7], which involves integration according to Hausdorff measure instead of Lebesgue measure used in the autocorrelation function, 
Eq.(2.1). In fact, we define the HMSF as follows:

$$
I^{s_{H}}(t)=\int_{x \in F} \mathcal{X}_{F}(x) \mathcal{X}_{F}(x+t) d \mathcal{H}^{s_{H}}(x),
$$

where $s_{H}$ is the Hausdorff dimension of the set $F$ [16]. Fig.5 shows the HMSF of the Cantor fractal set as well as of a geometrical multifractal set. The latter example is generated on the unit interval using two similitudes, with the scaling factors equal to $1 / 2$ and $1 / 4$, on left and right extremities, respectively. This two scale-Cantor set gives rise to what is called a geometrical multifractal $[17,18]$. Other typical examples can be found in $[6,7]$.
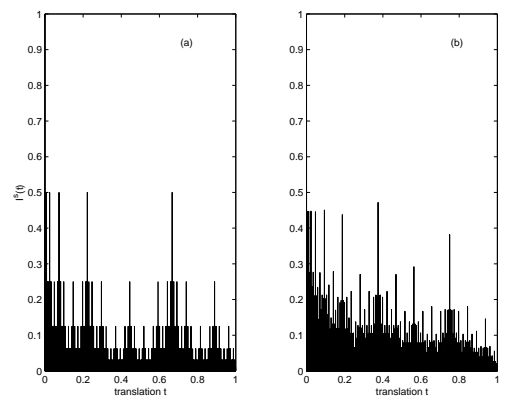

Fig. 5. The HMSF of the triadic Cantor set (left) and of the considered multifractal set (right).

\section{Distinguishing Sets of the Same Fractal Dimension Using HMSF}

The fractal dimension is used to quantify the complexity of a structure. However, there is a need to further set apart, in a quantitative way, different fractal structures sharing the same fractal dimension. In $[7,8]$, we have proposed the HMSF as a potential method to distinguish structures. By the way it is constructed, the HMSF contains information on two-point statistics relationship between parts in terms of their distances. This is the reason that the HMSF provides additional information on the organization of the structure (what is generically called lacunarity). We proposed two different ways to exploit the HMSF in order to distinguish between sets having the same fractal dimension. The first one is based on what we called the translation invariance based method (TIBM). The second one consists in comparing the measure values of this function for different sets at a fixed level. We call it the fixed level based method (FLBM). The latter one is necessary only when the first step in not enough. FLBM associates an index to sets which allows for their differentiation as well as for evaluation of their degree of homogeneity. For geometrical multifractals, peaks of the HMSF have variation of different heights. 
However, they still converge towards several level values, from which we can extract geometrical information related to the homogeneity of the structure. Further analysis in this direction will be done in a future publication.

\section{Estimation of the Hausdorff Dimension Using HMSF}

Our HMSF method is based on the Hausdorff measure and dimension, which are generally hard to be determined. This a priori knowledge can be an obstacle towards the application of the HMSF. We proposed a method that allows to approximate the Hausdorff measure and the Hausdorff dimension. The method we propose here does not rely on the exact Hausdorff measure definition but substitutes for it, an appropriate approximative quantity. Indeed, except for uniform Cantor-type sets whose HMSF can theoretically be determined $[6,7,9,16]$, it is necessary to use as a substitute, the measure spectrum function, which can be defined in the following way: given a fractal set $F$, we associate it with a family of covering sets $\left\{F_{i, n}\right\}$, where $n=0,1,2, \cdots$ and $i \in I_{n}$. The set $i \in I_{n}$ is the set of indexes whose corresponding intervals $F_{i, n}$ are nonempty. We also let the size $\left|F_{i, n}\right| \rightarrow 0$ when $n$ goes to infinity. If we write $F_{n}=\cup_{i \in I_{n}} F_{i, n}$, then $F=\cap_{n=1}^{\infty} F_{n}$. For the shift element $t$, the intersection $F_{n} \cap\left(F_{n}+t\right)=\cup F_{k, n}^{\prime}$, where $F_{k, n}^{\prime}$ corresponds to the intersection parts of $n$-th covering sets $\left\{F_{i, n}\right\}$ with their translates. Then, we can define the measure spectrum function as: $I_{n}^{s}(t)=\sum_{k}\left|F_{k, n}^{\prime}\right|^{s}$. This substitute is a generic approximation of the Hausdorff measure. When dealing with a given fractal set, one uses a coarse-graining process to practically represent the set at different resolutions. If we use a coarse-graining whose elements are all of size $\epsilon_{n}$ at the coarse-graining level $n$, we can define different quantities to approximate the Hausdorff measure [16]. A first approximating quantity is similar to the one involved in the box-counting dimension: $Q_{1}(n, s)=N(n) \epsilon_{n}^{s}$, where $N(n)$ is the number of nonempty boxes of coarse-graining elements of size $\epsilon_{n}$. A better approximating quantity is what we have previously named the adaptive coverings, noted $Q_{2}$ [16]. Indeed, we have successfully used this quantity to practically evaluate the Hausdorff dimension. $Q_{2}$ gathers into one element each group of joined coarse-graining elements, and redefines in this way another covering family (adaptive). It is usable when the condition that the maximum length of its adaptive covering goes to zero when the coarsegraining level $n$ goes to infinity [16]. We believe that for advanced needs of practical problems, this approximation exercise should be continued in order to reach better estimations. This quantity shows a transition property around its value at Hausdorff dimension $s_{H}$, similar to that exhibited by the Hausdorff measure. However, instead of relying upon the critical behaviour of $I_{s}(0)$ (which is related to the Hausdorff measure of the set) when $s$ is around $s_{H}$, we used the whole spectrum of measure function $I_{s}(t)$ for all values of $t$ varying from 0 to the total length of the set. In other words, it is a "global" measure phase transition criterion that we use instead of a transition criterion around a single point. This method provides an interesting alternative to the 
usual scaling forms involved in the box-counting dimension, where convergence problems are encountered.

\section{Uniqueness of HMSF}

The classical definition of autocorrelation function degenerates when applied to signals having support of zero Lebesgue measure. Though the power law expression can be used to quantify this degeneracy, the latter can have severe consequences since the usual autocorrelation function can be dependent on the dynamical process. In other words, one can get two different autocorrelation functions for the same signal if there exist two different dynamical processes, usually named cascades (also artificially obtained by coarse-graining), corresponding to this same final set. If we get interested in the asymptotic or infinite state of a physical phenomena, this degeneracy becomes a major drawback in signal characterization. Indeed, the coarse-graining procedure is the main way that we apply to represent a geometric set. However, this somehow artificial way can associate various dynamical systems to the same set, implying divergence in the autocorrelation power law exponent. Being concerned with this issue, the HMSF that we proposed seems to be a suitable form to drop the limitations of the classical autocorrelation function. Indeed, we showed that HMSF remedies to this situation and makes the characterization of the geometric information (at the static state) independent of the dynamic process involved[10].

\section{Conclusion}

In this paper, we established a statistical relationship between porosity and the autocorrelation function. This serves as a new definition, through the autocorrelation function, of the homogeneity and opens a new way to measure the departure of a given texture from its homogeneous ideal counterpart (the homogeneous image of the same porosity as the given texture). We also propose several new ways to investigate texture of porous media. These different approaches allow us to deal with a broad spectrum of texture complexity. Indeed, one cannot hope for an almighty solution for all textures. For various texture complexities, we have to develop different strategies aimed to different purposes. The new descriptions of texture obtained through these strategies define the various parameters which can be used for classification purposes or as reconstruction criteria. In fact, in this work, we have combined some largely used mathematical tools, namely the autocorrelation function and the Fourier transform, with more recent complexity analysis tools, i.e., the HMSF that we previously introduced along with the regularization dimension. This combination has been put in practice using computerized numerical means. This paper proposes some possible ways to probe the fine details in texture. 
However, for each of the proposed strategies, further work is going on theoretically and numerically. Parameter definitions can indeed be refined and more advanced algorithms have to be developed.

Acknowledgements. The authors would like to thank Professor Antoine Saucier for his valuable comments. This work has been supported by NSERC UFA, NSERC grant (RGPIN-227118) and MITACS, as well as by Faculté de Pharmacie, Univrsité de Montréal (fonds de démarrage) and by CRM (Centre de Recherches Mathématiques) group funds.

\section{References}

1. W. E. Wallace, C. M. Guttman (2002) J. Res. Natl. Inst. Stand. Technol 107: $1-17$

2. C. Allain, M. Cloitre (1987) Phys. Rev. A 36 no.12: 5751-5757

3. S. A. Bulgakov (1992) Phys. Rev. A 46 no.12:8024-8027

4. D. C. Champeney, (1973) Fourier Transforms and their Physical Applications. Academic Press, New York

5. L. S. Leibovitch (1998) Fractals and Chaos Samplified for the Life Sciences. Oxford University Press, New York

6. F. Nekka, J. Li (2002) Chaos, Solitons and Fractals 13, no.9:1807-1817

7. J. Li, F. Nekka (2004) Chaos, Solitons and Fractals 19, no.1:35-46

8. F. Nekka, J. Li (2004) Characterization of Fractal Structures Through a Hausdorff Measure Based Method. In: M. M. Novak (eds) Thinking in Pattern Fractals and Related Phenomena in Nature. World Scientific, Singapore

9. J. Li, F. Nekka (2003) Pattern Recognition Letters 24 Issue 15:2723-2730

10. J. Li and F. Nekka, Is the Classical Autocorrelation Function Appropriate for Complex Signals? - The Necessity of the Generalized Autocorrelation Function. Submitted to Int. J. of Chaos and Bifurcation 2004

11. F. Roueff, J. Levy-vehel (1998) A Regularization Approach to Fractional Dimension Estimation. In: M. M. Novak (eds) Fractals and Beyond - Complexities in the Sciences. World Scientific, Singapore

12. T.G. Smith Jr. et al. (1996) J. Neurosc. Methods 69:123-136

13. K. Zhao, Y. Deng, G. Q. Chen (2003) Biochemical Engineering Journal 16:115123

14. D. K. Umberger, J. D. Farmer (1985) Physical Review Letters 55 no.7:661-664

15. J. Li, C. Dubois, F. Nekka (2004) The Colligation of the Autocorrelation Function and the Regularization Dimension - A New Characterization of Porous Media. In: M. M. Novak (eds) Fractals 2004, Poster Abstracts, Complexity and Fractals in Nature, Vancouver

16. J. Li, A. Arneodo, F. Nekka (2004) CHAOS, An Interdisciplinary Journal of Nonlinear Science 14 no.4:1004-1017

17. J. Feder (1998) Fractals. Plenum Press, New York

18. T. Vicsek (1989) Fractal Growth Phenomena. World Scientific, Singapore 